\title{
Continuous vital sign monitoring after major abdominal surgery-Quantification of micro events
}

Duus, C. L.; Aasvang, E. K.; Olsen, Rasmus Munch; Sørensen, Helge Bjarup Dissing; Jørgensen, L. N.; Achiam, M. P.; Meyhoff, C. S.

Published in:

Acta Anaesthesiologica Scandinavica

Link to article, DOI:

10.1111/aas.13173

Publication date:

2018

Document Version

Peer reviewed version

Link back to DTU Orbit

Citation (APA):

Duus, C. L., Aasvang, E. K., Olsen, R. M., Sørensen, H. B. D., Jørgensen, L. N., Achiam, M. P., \& Meyhoff, C. S. (2018). Continuous vital sign monitoring after major abdominal surgery-Quantification of micro events. Acta Anaesthesiologica Scandinavica, 62, 1200-1208. https://doi.org/10.1111/aas.13173

\section{General rights}

Copyright and moral rights for the publications made accessible in the public portal are retained by the authors and/or other copyright owners and it is a condition of accessing publications that users recognise and abide by the legal requirements associated with these rights.

- Users may download and print one copy of any publication from the public portal for the purpose of private study or research.

- You may not further distribute the material or use it for any profit-making activity or commercial gain

- You may freely distribute the URL identifying the publication in the public portal 


\title{
Continuous vital sign monitoring after major abdominal surgery-Quantification of micro events
}

\author{
C. L. Duus ${ }^{1,2}$ (I) | E. K. Aasvang ${ }^{2}$ (D) | R. M. Olsen ${ }^{3}$ | H. B. D. Sørensen ${ }^{3}$ \\ L. N. Jørgensen ${ }^{4}$ | M. P. Achiam ${ }^{5}$ | C. S. Meyhoff ${ }^{1}$
}

${ }^{1}$ Department of Anaesthesia and Intensive Care, Bispebjerg and Frederiksberg Hospital, University of Copenhagen, Copenhagen, Denmark

${ }^{2}$ Department of Anaesthesiology, The Abdominal Centre, Rigshospitalet, University of Copenhagen, Copenhagen, Denmark

${ }^{3}$ Biomedical Engineering, Department of Electrical Engineering, Technical University of Denmark, Lyngby, Denmark

${ }^{4}$ Digestive Disease Center, Bispebjerg and Frederiksberg Hospital, University of Copenhagen, Copenhagen, Denmark

${ }^{5}$ Department of Surgical Gastroenterology, The Abdominal Centre, Rigshospitalet, University of Copenhagen, Copenhagen, Denmark

\section{Correspondence}

C. S. Meyhoff, Department of Anaesthesia and Intensive Care, Bispebjerg and Frederiksberg Hospital, Copenhagen, Denmark.

Email: christian.sahlholt.meyhoff@regionh.dk

Funding information

The Danish Cancer Society; Bispebjerg and Frederiksberg Hospital Research Council; The A.P. Møller Foundation
Introduction: Millions of patients undergo major abdominal surgery worldwide each year, and the post-operative phase carries a high risk of respiratory and circulatory complications. Standard ward observation of patients includes vital sign registration at regular intervals. Patients may deteriorate between measurements, and this may be detected by continuous monitoring. The aim of this study was to compare the number of micro events detected by continuous monitoring to those documented by the widely used standardized Early Warning Score (EWS).

Methods: Fifty patients were continuously monitored with peripheral arterial oxygen saturation $\left(\mathrm{SpO}_{2}\right)$, heart rate $(\mathrm{HR})$, and respiratory rate (RR) the first 4 days after major abdominal cancer surgery. EWS was monitored as routine practice. Number and duration of events were analyzed using Fisher's exact test and Wilcoxon rank sum test.

Results: Continuous monitoring detected a $\mathrm{SpO}_{2}<92 \%$ in $98 \%$ of patients vs $16 \%$ of patients detected by EWS $(P<.0001)$. Micro events of $\mathrm{SpO}_{2}<92 \%$ lasting longer than 60 minutes were found in $58 \%$ of patients by continuous monitoring vs $16 \%$ by the EWS $(P<.0001)$. Fifty-two percent of patients had micro events of $\mathrm{SpO}_{2}$ $<85 \%$ lasting longer than 10 minutes. Continuous monitoring found tachycardia in $60 \%$ of patients vs $6 \%$ by the EWS. Frequency of events for bradycardia, tachypnea, and bradypnea showed similar patterns.

Conclusion: Very low $\mathrm{SpO}_{2}$ and tachycardia in post-operative patients are common and under-diagnosed by the EWS. Continuous monitoring can discover these micro events and potentially contribute to earlier detection and, potentially, result in prevention of clinical complications.

\section{1 | INTRODUCTION}

Millions of patients undergo major abdominal surgical procedures annually. ${ }^{1}$ Post-operative complication rates are between 15\%-45\% depending on the surgical procedure, ${ }^{2,3}$ with prolonged in-hospital stay and Intensive Care Unit admission aside from the eminent discomfort and potential long-term disability. Among the most frequent complications are pulmonary and circulatory complications and it is estimated that 10 million patients experience myocardial injury after noncardiac surgery (MINS) each year. ${ }^{4,5}$ Prevention or early intervention of these complications would thus make a major difference in morbidity and mortality after abdominal surgery and cause economic savings. ${ }^{6-8}$ In an attempt to identify patients at risk of complications, standardized protocols like the Early Warning 
Score (EWS) have been implemented in several European countries. In the EWS, patients' vital signs (heart rate [HR], blood pressure, respiratory rate [RR], temperature, oxygen saturation, oxygen supplementation, and level of alertness) are monitored at pre-specified intervals starting at 12 hours and decreasing in case of abnormal vital signs (also called micro events).,10 During these long hours without observation patients might deteriorate without detection, as suggested by previous studies where $90 \%$ of cases with desaturation $<90 \%$ lasting longer than 60 minutes went undetected by routine ward rounds. Continuous monitoring may thus improve patient care by increasing the sensitivity and predictive accuracy of the detected deteriorations as well as detecting them sooner. ${ }^{11-13}$

The aim of this study was to compare the frequency of severe changes in post-operative cardiopulmonary physiological variables in patients monitored continuously as well as with the EWS following major abdominal cancer surgery. We hypothesized that continuous monitoring would detect severe micro events more frequently than EWS.

\section{2 | METHODS}

Approval was sought, but waived by the regional ethics committee as it was a purely observational study (protocol number 16038949). However, the study was approved by the Danish Data Protection Agency (2012-58-0004). The study was registered at www.ClinicalTri als.gov (NCT03102619) before inclusion began. Written informed consent was obtained from all participating patients.

\section{1 | Patient inclusion}

Fifty patients were enrolled between 23 March and 1 July 2017 at Bispebjerg Hospital and Rigshospitalet in Copenhagen, Denmark. Inclusion criteria were: age $\geq 65$ years, elective major abdominal cancer surgery with an estimated duration $>2$ hours, and preoperative sinus rhythm. Patients had to be eligible to receive optimized care according to the regimes on the given ward. Patients were excluded if they were unable to give informed consent or if they had a treatment limitation (such as a do-not-resuscitate-order).

\section{2 | Monitoring}

Isansys Lifetouch, Isansys Lifecare, Oxforshire, United Kingdom and Nonin WristOx 3150, Nonin Medical inc., Minnesota, USA were used. Lifetouch is a wireless patch with 2 ECG electrodes placed on the front left side of the thorax. The Lifetouch transmits information about HR and RR derived from a single lead ECG and sends this data via Bluetooth to a bedside gateway every minute as an average of the past 60 seconds. Nonin WristOx 3150 is a wearable pulse oximeter measuring peripheral arterial oxygen saturation $\left(\mathrm{SpO}_{2}\right)$ through standard digital measurement every second

\section{Editorial comment}

The Early Warning Score assesses vital signs intermittently. This study on major surgery patients found severe postoperative micro events for all measured parameters $\left(\mathrm{SpO}_{2}\right.$, $H R$, and RR) using continuous monitoring that were not always detected by the Early Warning Score.

and transfers data via Bluetooth to the gateway. Data from the pulse oximeter included raw data of values per second and a calculated average per minute of at least 5 reliable measurements. Alarms on all devices were switched off, screen light dimmed and nurses were instructed to make their own measurements for the EWS, following general practice, and not use the values from the research devices.

When patients were fit for discharge to the ward from the Post Anesthesia Care Unit (PACU) per the standardized discharge criteria, ${ }^{14}$ research personnel connected patients to the measuring devices. Patients were instructed not to disconnect unless they were attending personal hygiene or had diagnostic imaging done and these devices were to stay connected until discharge or for a maximum of 4 days. Thus, 50 patients had $\mathrm{SpO}_{2}, \mathrm{HR}$, and RR measured continuously for up to 4 days.

Nurses on the surgical ward observed and recorded the patients' vital signs according to the EWS algorithm per the clinical standard. ${ }^{10}$ Furthermore, we collected information on pain medication and epidural catheter.

Study personnel attended the participating patients at least once a day to change batteries in the devices and encourage patient compliance to reduce data loss.

\section{3 | Data analysis}

In the EWS algorithm, all measured values are converted to a score from 0 to 3 . The conversion into single parameter scores are presented in Table 1. The algorithm used in the Capital Region of Denmark requires physician assessment at a single parameter score of 3 or a cumulated score of 7 . To find micro events, data from the continuous monitoring as well as from the EWS were analyzed using threshold values from the EWS algorithm giving a single

TABLE 1 Single parameter scores according to the Early Warning Score

\begin{tabular}{lllll|} 
& 0 & 1 & 2 & 3 \\
\hline $\mathrm{HR}$ & $51-90$ & $41-50$ or $91-110$ & $111-130$ & $<41$ or $>130$ \\
$\mathrm{RR}$ & $12-20$ & $9-11$ & $21-24$ & $<9$ or $>24$ \\
$\mathrm{SpO}_{2}$ & $>95 \%$ & $94 \%-95 \%$ & $92 \%-93 \%$ & $<92 \%$ \\
\hline
\end{tabular}

$\mathrm{HR}$, heart rate in beats per minute; RR, respiratory rate in breaths per minute; $\mathrm{SpO}_{2}$ : peripheral arterial oxygen saturation. 
parameter score of 3 . The threshold values defining abnormal physiological parameters (micro events) were as follows: Hypoxemia was defined as $\mathrm{SpO}_{2}<92 \%$, tachycardia as $>130$ beats per minute, bradycardia as $<41$ beats per minute, tachypnea as $>24$ breaths per minute and bradypnea as $<9$ breaths per minute. In addition, the occurrence of severe hypoxemic episodes with $\mathrm{SpO}_{2}<85 \%$ and $80 \%$ were analyzed. All parameters from the two observation methods (continuous vs EWS) were compared regarding total number of occurrences, and duration of the individual events. The EWS provides an uncertain estimate of the duration of micro events because of the long interval in between measurements. The duration of an abnormal micro event in EWS was estimated as follows: The time between the observed abnormal value and the previous normal value divided by 2 , plus any consecutive time with abnormal values, plus the time between the observed abnormal value and the first subsequent normal value divided by 2 , thus assuming that the abnormal value started and ended midway between normal/abnormal values.

Artifacts were defined as: $\mathrm{SpO}_{2}$ : Any change larger than 4\% per second and all values less than $20 \%$ were considered artifacts. ${ }^{15}$ To calculate a reliable mean, we required at least 45 measurements per minute. All HR measurements $<20$ or $>250$ beats per minute and RR measurements $>60$ per minute were interpreted as artifacts. Missing data: $\mathrm{SpO}_{2}$ data were lost when the patient did not wear the device, when the battery was discharged, when the Bluetooth connection was lost, or if the bedside gateway was switched off. Data from the Lifetouch device were lost when the patch was loose or taken off.

In case of patients undergoing subsequent surgery or transfer to the ICU, data collection was interrupted until the patient returned to the ward.

\subsection{Statistical analysis}

Data were presented as number of patients with events (\%) or median and $5-95 \%$ range for duration of events. Mean differences are presented with $95 \%$ confidence intervals. For the statistical analysis, SAS version 9.4 (SAS Institute, Cary, NC, USA) was used. Association between categorical and continuous data were analyzed with the Fisher's exact test and Wilcoxon rank sum test respectively, and we considered $P<.05$ statistically significant.

\section{RESULTS}

The study included 50 patients for a total of 156.5 patient days of monitoring (on average 3.1 days per patient, Figure 1). Baseline characteristics are described in Table 2. EWS measurements were collected for all patients. A total of 693 EWS measurements were made which gave an average measurement interval of 5 hours and 25 minutes.

\subsection{Arterial oxygen saturation}

A total of 2529 hours of continuous $\mathrm{SpO}_{2}$ data were collected before removal of artifacts. After artifact removal (3\% of total $\mathrm{SpO}_{2}$ data), 2453 hours and 32 minutes remained. Average amount of data per patient was 49.1 hours corresponding to $65.3 \%$ of the total time (67.3\% before removal of artifacts).

One or more episodes of desaturation $<92 \%$ were found in $98 \%$ of the patients with continuous monitoring (Table 3 ) as compared to $16 \%$ with EWS $(P<.0001)$. The continuous monitoring found 109 events lasting longer than 60 minutes in 29 patients (58\%). Median

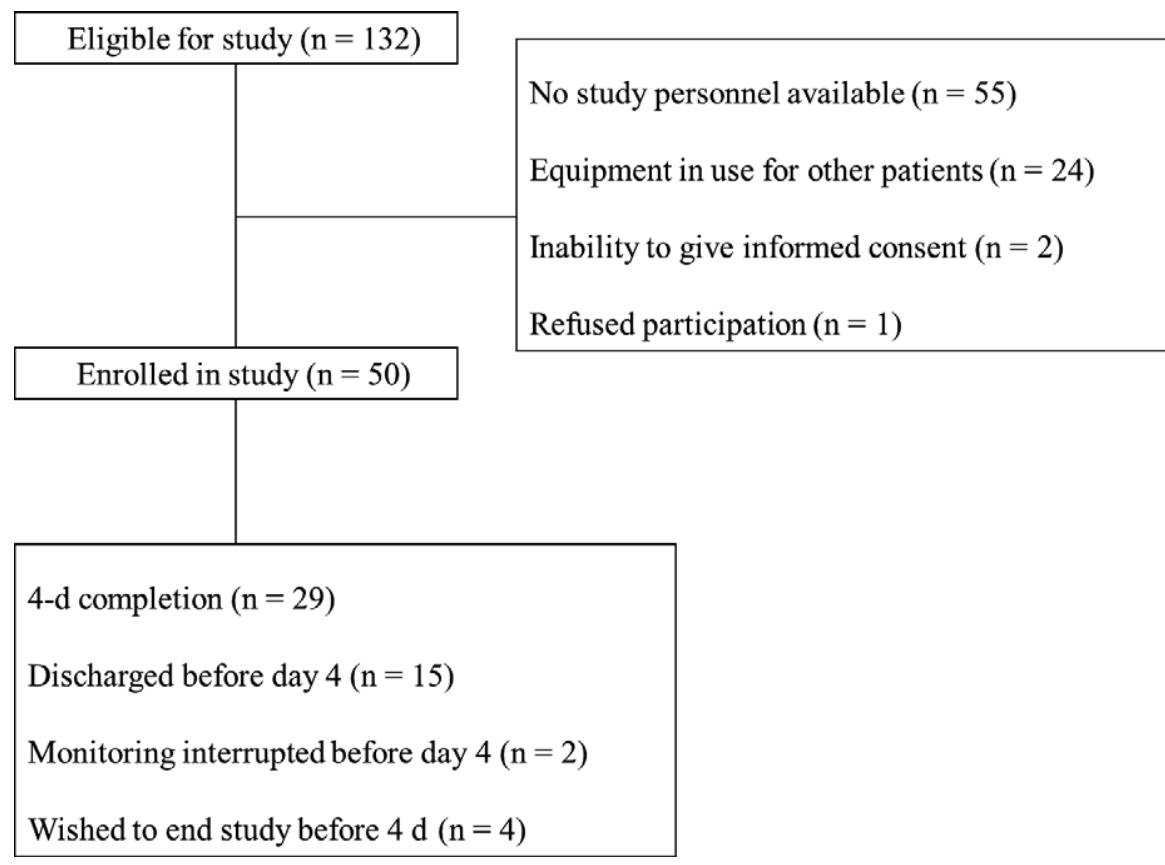

FIGURE 1 Trial flowchart 
TABLE 2 Baseline characteristics

\begin{tabular}{|c|c|}
\hline Parameter & $n=50$ \\
\hline Sex $M: F$ & $29: 21$ \\
\hline Age (y) & $71(65-81)$ \\
\hline Weight (kg) & $78(55-100)$ \\
\hline $\mathrm{BMI}\left(\mathrm{kg} / \mathrm{m}^{2}\right)$ & $26(19-35)$ \\
\hline \multicolumn{2}{|l|}{ ASA physical status score, No (\%) } \\
\hline ASA I & $4(8.0 \%)$ \\
\hline ASA II & $23(46 \%)$ \\
\hline ASA III & $23(46 \%)$ \\
\hline ASA IV & $0(0.0 \%)$ \\
\hline \multicolumn{2}{|l|}{ Medical history } \\
\hline Previous smoker & $20(40 \%)$ \\
\hline Current smoker & $12(24 \%)$ \\
\hline Alcohol consumption $^{a}$ & $8(16 \%)$ \\
\hline Previous stroke or TIA & $5(10 \%)$ \\
\hline Other neurological disease & $4(8.0 \%)$ \\
\hline Paroxysmal atrial fibrillation & $2(4.0 \%)$ \\
\hline Ischemic heart disease & $2(4.0 \%)$ \\
\hline Other cardiovascular disease & 7 (14\%) \\
\hline Chronic obstructive pulmonary disease & $5(10 \%)$ \\
\hline Asthma & $3(6.0 \%)$ \\
\hline Other pulmonary disease & $2(4.0 \%)$ \\
\hline Diabetes mellitus & $8(16 \%)$ \\
\hline Gastrointestinal disease & $5(10 \%)$ \\
\hline Other previous cancer diagnosis & $6(10 \%)$ \\
\hline Other disease & $7(14.0 \%)$ \\
\hline \multicolumn{2}{|l|}{ Type of surgery, $\mathrm{n}(\%)$} \\
\hline Colonic or rectal resection & 15 (30\%) \\
\hline Esophagectomy & 5 (10\%) \\
\hline Gastrectomy & $3(6.0 \%)$ \\
\hline Pancreatic resection & $6(12 \%)$ \\
\hline Pancreaticoduodenectomy & 7 (14\%) \\
\hline Liver resection & $8(16 \%)$ \\
\hline Explorative laparotomy & $6(12 \%)$ \\
\hline Epidural catheter & $33(66 \%)$ \\
\hline Post-operative opioids & $18(36 \%)$ \\
\hline Post-operative opioid consumption (mg) & $40(20-160)$ \\
\hline
\end{tabular}

BMI, body mass index; TIA, transient ischemic attack.

Values are number (percentage) or median (5\%-95\% range). Opioid consumption is reported as intravenous morphine equivalents and only for the receiving patients.

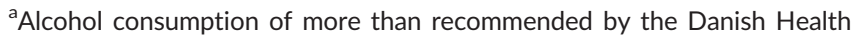
Authority, which is $24 \mathrm{~g} / \mathrm{d}$ for men or $12 \mathrm{~g} / \mathrm{d}$ for women.

number of events lasting longer than 60 minutes per patient was 1 (5\%-95\% range 0-7 events). Median hypoxemic time per patient was 435.5 minutes (5\%-95\% range 16-1801 minutes).

The mean difference between hypoxemic time according to the continuous monitoring and to the EWS was 626 minutes $(95 \% \mathrm{Cl}$
420-833 minutes). Five of the desaturation events caught by the EWS was not found by the continuous monitoring, due to missing data from these periods of time.

Figure 2 illustrates the distribution of desaturation $\left(\mathrm{SpO}_{2}<92 \%\right)$ on days after surgery as well as time of day. Desaturation occurred mainly on the second and third post-operative day and the distribution of total hypoxemic time was as follows: $34.9 \%$ of total hypoxemic time was between 00 and 08, 27.4\% between 08 and 16, and $37.7 \%$ between 16 and 24 .

Hypoxemia with $\mathrm{SpO}_{2}<85 \%$ was found in $88 \%$ of the patients with continuous monitoring, whereas the EWS only detected $\mathrm{SpO}_{2}$ $<85 \%$ in 2 patients (4\%). Fifty-two percent of patients had at least one episode of $\mathrm{SpO}_{2}<85 \%$ lasting more than 10 minutes. Mean difference in time with $\mathrm{SpO}_{2}<85 \%$ when monitored continuously vs by the EWS was 43.6 minutes. The continuous monitoring found that $56 \%$ of the patients desaturated to $<80 \%$ at some point during the study while the EWS only found this in $2 \%$ of patients $(P<.0001)$.

\subsection{Heart rate}

The accumulated time of obtained continuous HR data was 3353 hours. After removal of artifacts (0.03\% of recorded time), 3352 hours were available. Continuous HR and RR data were obtained $89.2 \%$ of the total time and the average time per patient after artifact removal was 67 hours and 5 minutes.

Sixty percent of the patients were found to be tachycardic at some point during the study by the continuous monitoring, however, tachycardia was only recorded in $6 \%$ of patients $(P<.0001)$ by the EWS. Continuous monitoring detected bradycardia in $20 \%$ of the patients and $4 \%$ were bradycardic for more than 60 minutes. No bradycardic episodes were reported by the EWS.

\section{3 | Respiratory rate}

Total monitored time for continuous RR was 3350 hours and no artifacts were found. Tachypnea was detected in $74 \%$ of the patients by the continuous monitoring and median duration of tachypnea was 8.0 minutes (5\%-95\% range 0-189 minutes). The EWS discovered 10 events of tachypnea out of which one was at a time without continuously monitoring switched on. In seven events of tachypnea registered in the EWS, a normal respiration rate was recorded by the continuous monitoring.

The continuous monitoring detected bradypnea in $64 \%$ of the patients. The EWS found bradypnea in one patient (2\%). This event was not detected by the continuous monitoring. The median duration of bradypnea according to the EWS was 2.0 minutes (mean difference 40.6 minutes, $95 \% \mathrm{Cl}$ 12.1-69.2 minutes).

For all the measured parameters continuous monitoring found events in more patients than the EWS. Also, differences in duration of micro events for the individual patient were discovered. The EWS monitoring estimate resulted in long durations due to the intervals between measurements, but only in few patients, whereas 
TABLE 3 Cardiopulmonary micro events after major abdominal cancer surgery

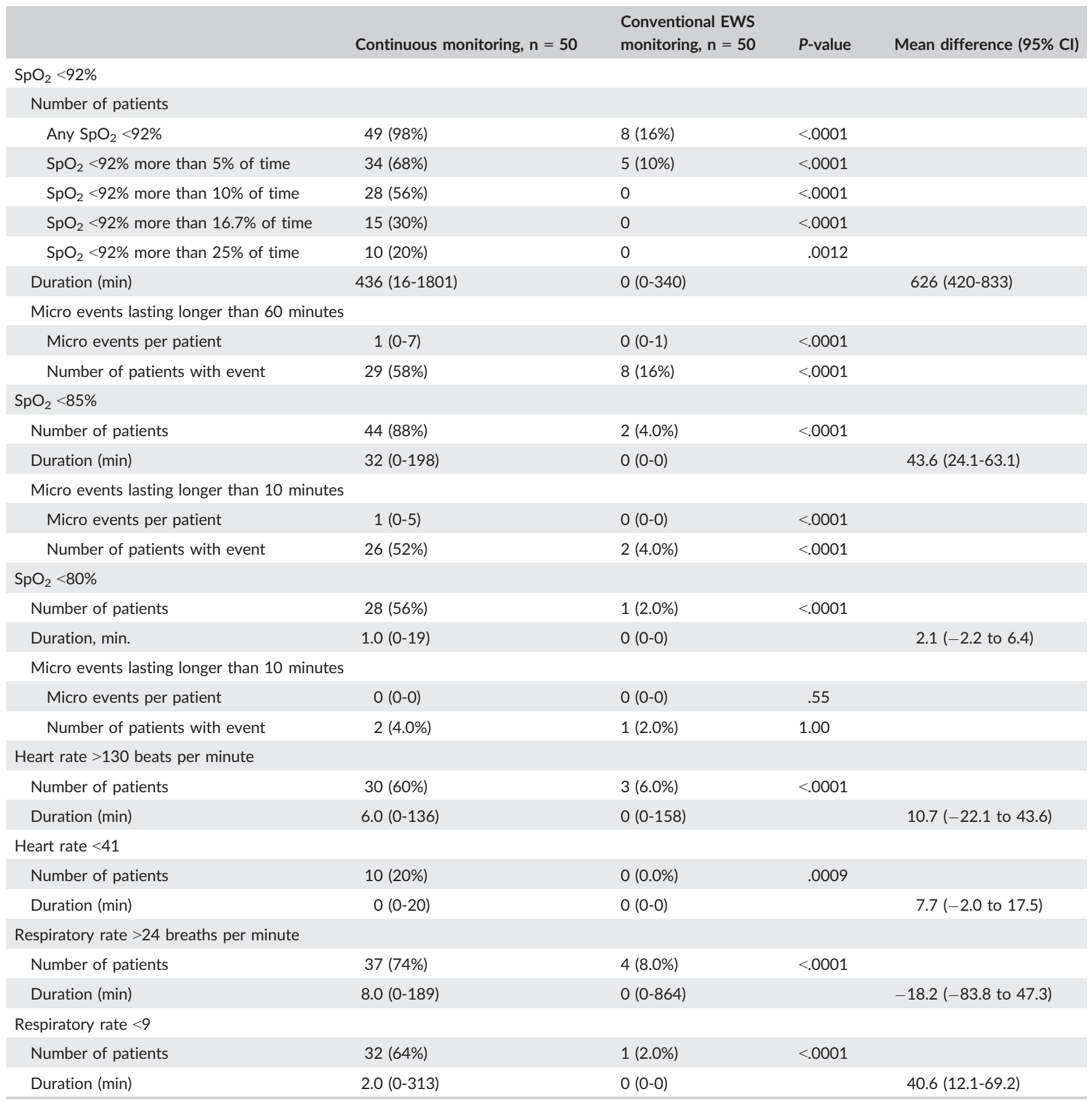

Values are number (percentage) or median (5\%-95\% range). Duration of each event is calculated as average cumulative duration among all included patients.

continuous monitoring detected deteriorations of varying duration and in many patients (Figure $3 \mathrm{~A}-\mathrm{C}$ ).

\section{DISCUSSION}

Our study found clinically and statistically significant discrepancies between the EWS-recorded abnormal vital parameters and those registered by continuous monitoring in patients after major abdominal cancer surgery. Hypoxemic micro events of $\mathrm{SpO}_{2}<92 \%$ were found in $98 \%$ of patients with continuous monitoring compared to only $16 \%$ found by the EWS. These severe micro events were often of considerable duration, and severe desaturations $\left(\mathrm{SpO}_{2}\right.$ $<85 \%$ ) lasting more than 10 minutes occurred in more than half of patients.

We were able to examine $\mathrm{SpO}_{2}, \mathrm{HR}$, as well as RR continuously for 4 days in high-risk patients. This provided us with a substantial amount of data despite a limited sample size of 50 patients and 


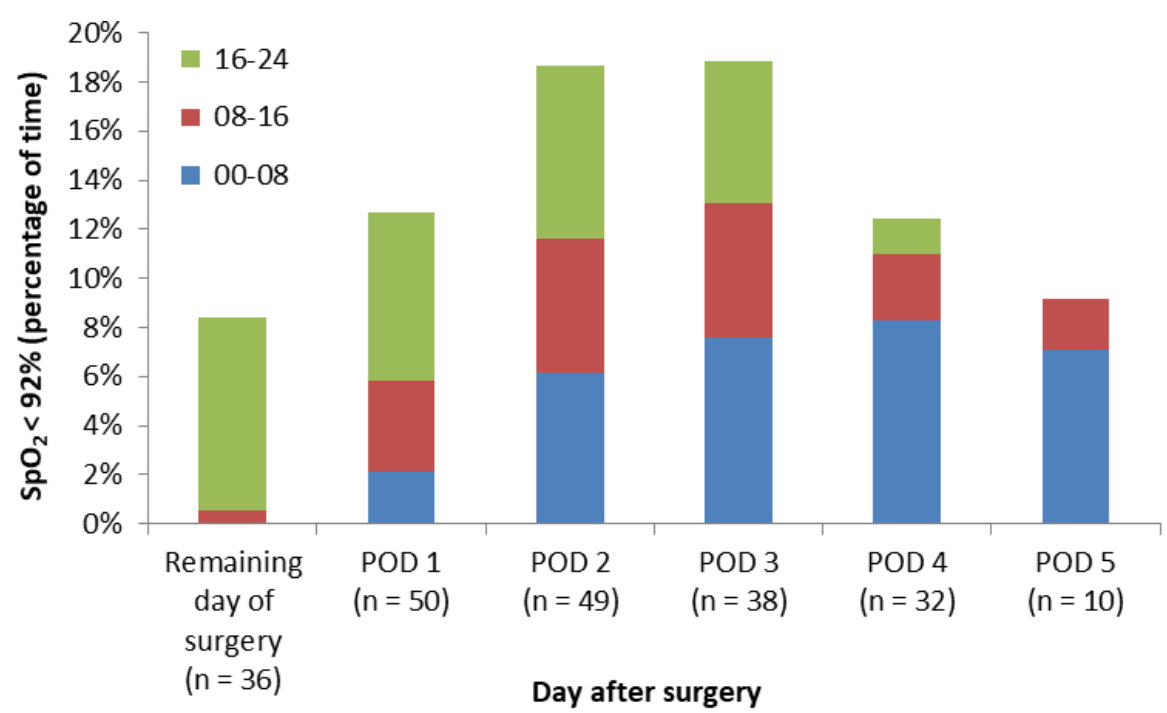

FIGURE 2 Percentage $\mathrm{SpO}_{2}<92 \%$ out of total monitored time per day. POD, post-operative day; n, number of patients monitored that day; blue color, desaturation at night time (00:00 to 08:00), red color, desaturation at day time (08:00 to 16:00); green color, desaturation at evening time (16:00 to $24: 00)$

allowed us to detect statistically and clinically significant differences in detected micro events. A post-hoc power analysis confirms that by including 50 patients, we had power for detecting the observed difference in $\mathrm{SpO}_{2}<92 \%$ of more than $95 \%$ with a level of significance of $5 \%$ and at least a power of $80 \%$ for the remaining micro events.

However, $\mathrm{SpO}_{2}$ data collection was only achieved for $65.3 \%$ of time, and adverse episodes during the non-recorded periods may not have been identified, which means that the current observations may be lower than if we had had complete $\mathrm{SpO}_{2}$ data. Since we calculated the hypoxemic time as percentage of the total observation time and not the recorded time (assuming patients to be normoxic during the missing data periods), our results represent minimum numbers. Missing $\mathrm{SpO}_{2}$ data are comparable to a recent study by Sun et al ${ }^{16}$ in which only 833 of 1339 measured patients had sufficient $\mathrm{SpO}_{2}$ data. Technical difficulties (battery power, Bluetooth connection unavailable or bedside gateway switched off) were the main reasons for loss of data, but patient compliance was also challenged, if patients had delirium or felt physically restricted by the devices. Last, data were also missing during mobilization and personal hygiene as part of routine accelerated care. Due to missing $\mathrm{SpO}_{2}$ data, five of the desaturation micro events detected by the EWS were not found by the continuous monitoring, however, continuous monitoring discovered more than 3000 micro events not detected by the EWS.

Similar to our study, technological advances now allow for continuous monitoring of vital parameters with so-called wear-and-forget devices to assess the usability and compare them to conventional measurements. ${ }^{17-19}$

Estimating the arterial oxygen saturation from pulse oximetry has a number of limitations. Performance of the pulse oximeter $\left(\mathrm{SpO}_{2}\right)$ to reflect arterial oxygenation is subject to biological variability as well as sensor variability. ${ }^{20}$ Sensor accuracy is primarily determined by the wavelength of the LED in the sensor, which varies between manufacturers. The Nonin WristOx in our study is FDA and CE marked and calibrated. Biological variability is mostly pronounced in situations with low peripheral perfusion (eg vascular contraction from pain, low temperature, hypotension etc.) or movement of the pulse oximeter, ${ }^{20}$ and in those situations, continuous monitoring may report lower $\mathrm{SpO}_{2}$ than measurements with $\mathrm{EWS}$, where the nurses have the opportunity to fit the pulse oximeter to the highest possible recording of $\mathrm{SpO}_{2}$. Taenzer et al ${ }^{19}$ found discrepancy between nurse $\mathrm{SpO}_{2}$ recordings and electronic recordings, and nurse measurements were on average $6.5 \%$ (95\% Cl 4.0\%-9.0\%) higher than electronic recordings. Another study found differences between continuous monitoring and nurse recordings on the RR with both fewer and more counts by nurses than detected electronically. ${ }^{18}$

High frequency of desaturation has previously been described with continuous monitoring. Sun et al. investigated the incidence of $\mathrm{SpO}_{2}<90 \%$ in unselected patients undergoing non-cardiac surgery in the 48 hours following discharge from PACU, and found desaturations for more than $16.7 \%$ of the observation time in $21 \%$ of included patients. ${ }^{16}$ We found $30 \%$ of patients to have $\mathrm{SpO}_{2}<92 \%$ more than $16.7 \%$ of the time. Our higher number may be related to the higher-risk surgery and the different $\mathrm{SpO}_{2}$ threshold. Regarding duration of events, Sun et al. found events of $\mathrm{SpO}_{2}<90 \%$ lasting longer than 60 minutes in $37 \%$ of patients, whereas we found events of $\mathrm{SpO}_{2}<92 \%$ with a duration of more than 60 minutes in $58 \%$ of patients. Similar to our results, Sun et al. found a lower detection of desaturation by the nurses than by continuous monitoring as only $5 \%$ of patients had any hypoxemia according to nursing flow sheets. We found the same discrepancy for HR and RR, and the lesser detection of these vital parameters with the EWS has not been described previously.

Night time $\mathrm{SpO}_{2}$ was measured continuously by Gögenur et al., ${ }^{21}$ and $\mathrm{SpO}_{2}<90 \%$ was detected in $90 \%$ of recorded patient nights and more than 2400 episodes of hypoxemia were reported. However, 

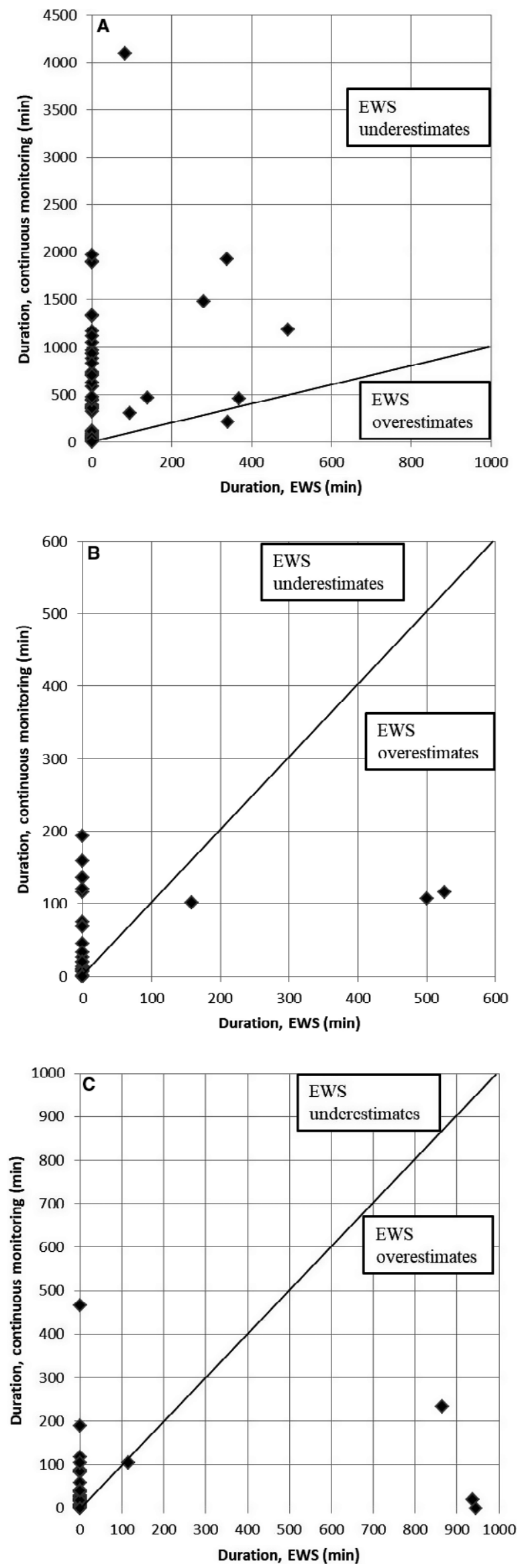

FIGURE 3 Duration of measured micro events in minutes per patient according to continuous monitoring and EWS, respectively. A: Duration of $\mathrm{SpO}_{2}<92 \%$, B: Duration of tachycardia (HR $>130 \mathrm{bpm}$ ), C: Duration of tachypnea (RR $>24 \mathrm{bpm}$ ). $\mathrm{SpO}_{2}$, arterial oxygen saturation; EWS, Early Warning Score

the study did not report duration or total time of recorded hypoxemia which is an important factor as long intervals of low $\mathrm{SpO}_{2}$ have been suggested to be involved in the pathogenesis of several common post-operative complications. ${ }^{22}$

According to the EWS algorithm all $\mathrm{SpO}_{2}$ values $<92 \%$ requires the nurse to contact a physician. ${ }^{10}$ It is therefore of clinical significance that this appears to occur in almost all post-operative patients in our study. As this high occurrence of unreported hypoxemia has been described in previous studies, the post-operative regimes on the wards include supplementary oxygen. Patients included in our study often had nasal catheter with oxygen; $96 \%$ had supplementary oxygen during the first post-operative day, $76 \%$ during the second day, $58 \%$ during the third day and $34 \%$ during the 4 th day. However this evidently did not eliminate the exposure to hypoxemia. Post-operative imbalance of oxygenation (either hypoxemia or supplemental oxygen resulting in hyperoxia) has been linked to several post-operative cerebral, pulmonary and cardiac complications, and surgical site infections. ${ }^{21,23-25}$ However, our study was not powered to detect differences in clinical outcomes, but these must be considered in future larger trials to substantiate the effect of continuous monitoring and advocate for the initial increased expenses and continued research. ${ }^{17}$

The EWS is commonly used in European countries for postoperative observation and is connected to an escalation protocol for more intensive measurements and potentially a rapid response team, if vital parameters are affected. ${ }^{10,26}$ However, this study raises important questions regarding the sensitivity of this method, because the escalation protocol may not be activated if monitoring is not performed continuously. ${ }^{27}$ Although differences in results for HR and $\mathrm{RR}$ were not as prominent as for $\mathrm{SpO}_{2}$, most findings were statistically significant in terms of more sensitive detection of events with continuous monitoring. This emphasizes that patients' vital signs vary in the days following major surgery, and that one normal measurement in the morning does not necessarily reflect normal values for the remaining day. Continuous detection of micro events may lead to the de novo identification of new types of vital sign aberrations, and this may in turn modify thresholds for clinical interventions or indicate novel treatment pathways with the potential to be more effective in keeping improvements in vital signs stable.

With continuous monitoring, we found $64 \%$ of patients to be bradypneic ( $R R<9$ breaths per minute) at some point during the first four post-operative days, while the EWS only found this in $2 \%$. Patients are often awake and interacting with the nurses during the EWS measurement. This may partly explain why the EWS rarely finds bradypnea, as the RR is often reduced during sleep. However, we did not analyze whether bradypnea was mainly found during sleep or the relation to desaturation. 
Seven episodes of tachypnea ( $R R>24$ breaths per minute) were registered by the EWS at times where the continuous monitoring did not find tachypnea. This may be due to the difference in the measuring methods as the continuous patch monitoring analyses changes in the amplitude of the R peak in the ECG, whereas the nurses count the number of breaths manually for the EWS. ${ }^{26}$ Most reliable method is yet to be determined, and as previously stated a discrepancy has earlier been observed between these two methods as Weenk et al ${ }^{18}$ found both higher and lower counts of RR by continuous monitoring compared to standard measuring.

An advantage of the EWS is the contact between the nurse and the patient, which is an opportunity for the nurse to observe the patient clinically. However, this physical contact also has its disadvantages since nurses have to wake up patients late at night or early in the morning to perform measurements. This may result in situations where the reported vital signs in the EWS do not reflect physiology during night time. We collected several examples of patients with low $\mathrm{SpO}_{2}$ in the minutes before EWS measurement which subsequently turned normal or near-to-normal for a few minutes where after it returned to lower levels. Thus, the EWS potentially reflects the physiological capacity rather than the actual status and function. The EWS hereby provides false security for nurses and physicians who might not notice the deterioration of the patient. This potential problem could be diminished with continuous monitoring. In one extreme case, a patient was hypoxemic $72.7 \%$ of the time and the desaturation of this patient was not at any time discovered by the EWS. Severely low $\mathrm{SpO}_{2}$ may inflict organ damage such as in the brain and heart. Our study thus indicates that continuous monitoring theoretically can be beneficial to post-operative organ function, but the exact threshold for hypoxia-induced organ damage is not yet established.

\section{5 | CONCLUSION}

We found significantly more severe micro events of all measured parameters $\left(\mathrm{SpO}_{2}, \mathrm{HR}\right.$, and $\left.\mathrm{RR}\right)$ using continuous monitoring than reported by the EWS. Prolonged hypoxemia of potential clinical importance was common in this high-risk patient group the days following major abdominal cancer surgery and severe long-lasting hypoxemic episodes were seen in more than half of patients. Future studies must evaluate the clinical effect of continuous monitoring compared to standardized interval based observation and intervention in terms of the thresholds and durations of vital sign aberrations that are most predictive of adverse outcome as well as the clinical interventions that are most likely to make a positive difference.

\section{ACKNOWLEDGEMENTS}

C. L. D.: Received funding from Bispebjerg and Frederiksberg Hospital Research Council. R. M. O.: Received funding from The Danish Cancer Society. Other authors: Departmental funding only. The A. P. Møller Foundation funded research equipment.

\section{CONFLICT OF INTEREST}

C. S. M. reports direct and indirect research funding from Ferring Pharmaceuticals, Merck Sharp \& Dohme Corp., and Boehringer Ingelheim outside the submitted work. Other authors report none.

\section{ORCID}

C. L. Duus (iD http://orcid.org/0000-0001-9893-5573

E. K. Aasvang (D) http://orcid.org/0000-0002-7131-2461

L. N. Jørgensen (iD http://orcid.org/0000-0001-7465-5374

C. S. Meyhoff (iD http://orcid.org/0000-0002-4885-4609

\section{REFERENCES}

1. Rose J, Weiser TG, Hider P, Wilson L, Gruen RL, Bickler SW. Estimated need for surgery worldwide based on prevalence of diseases: a modelling strategy for the WHO Global Health Estimate. Lancet Glob Health. 2015;3(Suppl 2):S13-S20.

2. Mokart D, Giaoui E, Barbier L, et al. Postoperative sepsis in cancer patients undergoing major elective digestive surgery is associated with increased long-term mortality. J Crit Care. 2016;31:48-53.

3. Markar S, Gronnier C, Duhamel A, et al. Pattern of postoperative mortality after esophageal cancer resection according to center volume: results from a large European multicenter study. Ann Surg Oncol. 2015;22:2615-2623.

4. Ball L, Battaglini D, Pelosi P. Postoperative respiratory disorders. Curr Opin Crit Care. 2016;22:379-385.

5. Devereaux PJ, Biccard BM, Sigamani A, et al. Association of postoperative high-sensitivity troponin levels with myocardial injury and 30-day mortality among patients undergoing noncardiac surgery. JAMA. 2017;317:1642-1651.

6. Govaert JA, Fiocco M, van Dijk WA, et al.; Dutch Value Based Healthcare Study Group. Costs of complications after colorectal cancer surgery in the Netherlands: building the business case for hospitals. Eur J Surg Oncol. 2015;41:1059-1067.

7. Vonlanthen R, Slankamenac K, Breitenstein S, et al. The impact of complications on costs of major surgical procedures: a cost analysis of 1200 patients. Ann Surg. 2011;254:907-913.

8. Vallribera Valls F, Landi F, Espin Basany E, et al. Laparoscopyassisted versus open colectomy for treatment of colon cancer in the elderly: morbidity and mortality outcomes in 545 patients. Surg Endosc. 2014;28:3373-3378.

9. Petersen JA, Antonsen K, Rasmussen LS. Frequency of Early Warning Score assessment and clinical deterioration in hospitalized patients: a randomized trial. Resuscitation. 2016;101:91-96.

10. Royal College of Physicians. National Early Warning Score (NEWS): Standardising the Assessment of Acute Illness Severity in the NHS. Report of a working party. London: RCP; 2012.

11. Taenzer AH, Pyke JB, McGrath SP, Blike GT. Impact of pulse oximetry surveillance on rescue events and intensive care unit transfers: a before-and-after concurrence study. Anesthesiology. 2010;112:282287.

12. Moss TJ, Clark MT, Calland JF, et al. Cardiorespiratory dynamics measured from continuous ECG monitoring improves detection of deterioration in acute care patients: a retrospective cohort study. PLoS ONE. 2017;12:e0181448. https://doi.org/10.1371/journal. pone. 0181448

13. Haahr-Raunkjaer C, Meyhoff CS, Sorensen HBD, Olsen RM, Aasvang EK. Technological aided assessment of the acutely ill patient - The case of postoperative complications. Eur J Intern Med. 2017;45:4145. 
14. Aldrete JA. The post-anesthesia recovery score revisited. J Clin Anesth. 1995;7:89-91.

15. Magalang UJ, Dmochowski J, Veeramachaneni S, et al. Prediction of the apnea-hypopnea index from overnight pulse oximetry. Chest. 2003;124:1694-1701.

16. Sun Z, Sessler DI, Dalton JE, et al. Postoperative hypoxemia is common and persistent: a prospective blinded observational study. Anesth Analg. 2015;121:709-715.

17. Michard F, Gan TJ, Kehlet H. Digital innovations and emerging technologies for enhanced recovery programmes. $\mathrm{Br} J$ Anaesth. 2017;119:31-39.

18. Weenk M, van Goor H, Frietman B, et al. Continuous monitoring of vital signs using wearable devices on the general ward: pilot study. JMIR Mhealth Uhealth 2017;5:e91. Jul 05. https://doi.org/10.2196/ mhealth.7208

19. Taenzer AH, Pyke J, Herrick MD, Dodds TM, McGrath SP. A comparison of oxygen saturation data in inpatients with low oxygen saturation using automated continuous monitoring and intermittent manual data charting. Anesth Analg. 2014;118:326-331.

20. Louie A, Feiner JR, Bickler PE, Rhodes L, Bernstein M, Lucero J. Four types of pulse oximeters accurately detect hypoxia during low perfusion and motion. Anesthesiology. 2018;128:520-530.

21. Gogenur I, Rosenberg-Adamsen S, Lie C, Carstensen M, Rasmussen $\mathrm{V}$, Rosenberg J. Relationship between nocturnal hypoxaemia, tachycardia and myocardial ischaemia after major abdominal surgery. Br J Anaesth. 2004;93:333-338.

22. Habre W, Petak F. Perioperative use of oxygen: variabilities across age. Br J Anaesth 2014;113(Suppl 2):ii26-ii36.
23. Meyhoff CS, Jorgensen LN, Wetterslev J, Christensen KB, Rasmussen LS. Increased long-term mortality after a high perioperative inspiratory oxygen fraction during abdominal surgery: follow-up of a randomized clinical trial. Anesth Analg. 2012;115:849-854.

24. Fonnes S, Gogenur I, Sondergaard ES, et al. Perioperative hyperoxia - long-term impact on cardiovascular complications after abdominal surgery, a post hoc analysis of the PROXI trial. Int $J$ Cardiol. 2016;215:238-243.

25. Staehr-Rye AK, Meyhoff CS, Scheffenbichler FT, et al. High intraoperative inspiratory oxygen fraction and risk of major respiratory complications. Br J Anaesth. 2017;119:140-149.

26. Petersen JA, Mackel R, Antonsen K, Rasmussen LS. Serious adverse events in a hospital using early warning score - what went wrong? Resuscitation. 2014;85:1699-1703.

27. Subbe CP, Kruger M, Rutherford P, Gemmel L. Validation of a modified Early Warning Score in medical admissions. QJM. 2001;94:521-526.

How to cite this article: Duus CL, Aasvang EK, Olsen RM, et al. Continuous vital sign monitoring after major abdominal surgery-Quantification of micro events. Acta Anaesthesiol Scand. 2018;00:1-9. https://doi.org/10.1111/aas.13173 